\title{
Molecular dynamics simulations of ballistic annihilation
}

\author{
Adam Lipowski, ${ }^{1}$ Dorota Lipowska, ${ }^{2}$ and António L. Ferreira ${ }^{3}$ \\ ${ }^{1}$ Faculty of Physics, Adam Mickiewicz University, 61-614 Poznań, Poland \\ ${ }^{2}$ Institute of Linguistics, Adam Mickiewicz University, 60-371 Poznań, Poland \\ ${ }^{3}$ Department of Physics, University of Aveiro, 3810 Aveiro, Portugal
}

\begin{abstract}
Using event-driven molecular dynamics we study one- and two-dimensional ballistic annihilation. We estimate exponents $\xi$ and $\gamma$ that describe the long-time decay of the number of particles $(n(t) \sim$ $\left.t^{-\xi}\right)$ and of their typical velocity $\left(v(t) \sim t^{-\gamma}\right)$. To a good accuracy our results confirm the scaling relation $\xi+\gamma=1$. In the two-dimensional case our results are in a good agreement with those obtained from the Boltzmann kinetic theory.
\end{abstract}

PACS numbers: $82.20 . \mathrm{Nk}$

Kinetics of reacting particle systems is recently intensively studied. Such systems model a wealth of different phenomena and in addition offer an important testing ground for nonequilibrium statistical mechanics. In lattice formulation such systems are much easier to analyze and in some cases even exact results could be obtained. More realistic, off-lattice (ballistic) versions are at the same time much more difficult to examine, but even here some analytical insight is possible [1].

Despite being one of the simplest reacting particle systems, single-species ballistic annihilation, $A+A \rightarrow 0$, so far evaded exact solution. Consequently, our understanding of the kinetics of this process comes mainly from approximate methods. To describe ballistic annihilation one usually calculates density of particles $n(t)$ and their typical velocity $v(t)$ defined using the second moment of the time-dependent velocity distribution $f(\boldsymbol{v}, t)$

$$
v(t)^{2}=\frac{1}{n(t)} \int v^{2} f(\boldsymbol{v}, t) d \boldsymbol{v}
$$

Of particular interest are then the exponents $\xi$ and $\gamma$ that describe the asymptotic decay of these quantities

$$
n(t) \sim t^{-\xi}, \quad v(t) \sim t^{-\gamma}
$$

A simple power counting or scaling arguments can be used to show that

$$
\xi+\gamma=1
$$

Although a rigorous justification of this relation is missing, Piasecki et al. [5] analysing BBGKY-like hierarchy presented convincing arguments supporting relation (3).

A lot of efforts was made to calculate $\xi$ and $\gamma$ 2, 3, 4, 5, 6, 7]. However, it would be desirable to obtain more accurate estimations of these exponents since it could increase our understanding in this field. For example, some arguments suggest [5] that in dimensions $d>1$ Boltzmann kinetic theory provides an accurate description of such a process. However, a direct verification of this hypothesis, using molecular dynamics simulations, is not yet fully completed mainly due to too small number of particles that were taken into account. Let us also notice that current estimations of $\xi$ for $d=1,2$ and 3 are surprisingly close to the result obtained in the so-called reaction-controlled limit $(\xi=4 d /(4 d+1))$ [4]. Further check whether this exponent could be given as such a simple fraction would be desirable. Let us notice that a closely related process, namely ballistic aggregation, is solvable in $d=1$ and some of its exponents are indeed simple fractions [8].

In the present paper we describe results of extensive molecular dynamics simulations of ballistic annihilation in $d=1$ and 2. Since in such a process particles interact (i.e., annihilate) only upon collision efficient simulations can be made with the use of the so-called event-driven dynamics [9]. One of the difficulties in this technique is the search for the time of the nearest collision. Most efficient algorithms to locate such an event arrange data on the heap tree 10]. For event-driven simulations for $d>1$ systems it is also essential to include sectorization and search for a collision partner only within a given sector and its nearest neighbours. Heap tree searching and sectorization were already used in event-driven dynamics of various hard-core or granular systems 11, 12, 13] but to our knowledge not in the ballistic annihilation. Since it substantially improves the numerical performance, our method implements these techniques. The fact that at the collision particles annihilate, simplifies the algorithm comparing to the non-annihilating systems. This is because there is no need to examine post-collision events of colliding particles. In the following we discuss obtained numerical results.

In the $d=1$ case we place $N$ point-like particles on a line interval of length $L=N$. Such a choice keeps the initial density of particles $n=N / L$ constant (and equal to unity) and that will allow us the comparison of results for different values of $N$. Initially velocities have a distribution $f(\boldsymbol{v}, t=0)$ that either will be gaussian or it will have a $\boldsymbol{v}=\mathbf{0}$ singularity $f(\boldsymbol{v}, t=0) \sim|\boldsymbol{v}|^{-\mu}$ with a characteristic exponent $\mu$. Periodic boundary conditions are used. We measured the density of particles $n(t)$ at time $t$ and their typical velocity $v(t)$ defined using Eq. (11). To estimate the exponents $\xi$ and $\gamma$ the system must reach the asymptotic regime. However, for finite $N$ the long-time behaviour is affected by poor statistics as well as by finite-size effects and that hinders precise determination of $\xi$ and $\gamma$. To take these effects into ac- 


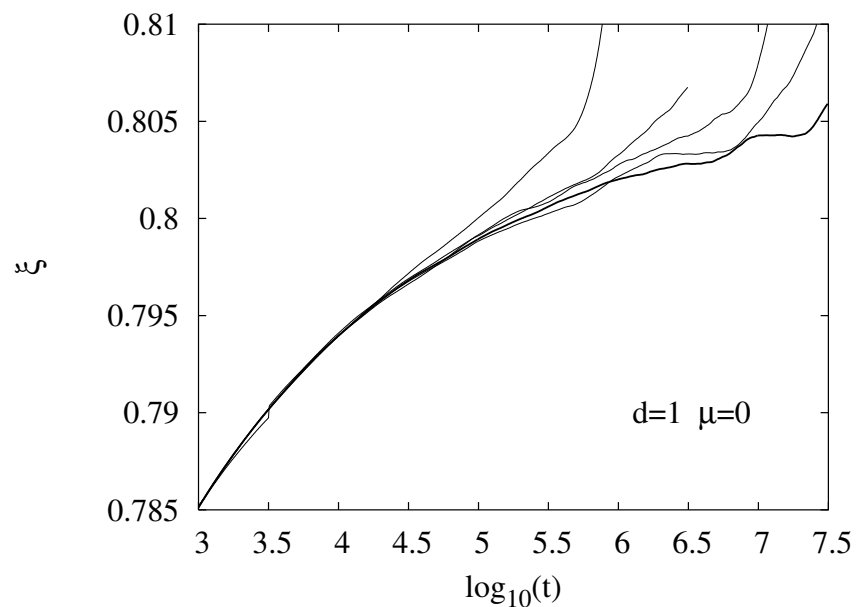

FIG. 1: The exponent $\xi$ as a function of $t$ for $N=10^{6}, 5$. $10^{6}, 10^{7}, 2 \cdot 10^{7}$, and $3 \cdot 10^{7}$ (thick line). Initially, velocities have a gaussian distribution $(\mu=0)$.

count we calculated time-dependent exponents $\xi(t)$ and $\gamma(t)$ using results (i.e., $n(t)$ and $v(t))$ spreading over one decade around a given value of $t$.

For the gaussian initial velocity distribution our results are shown in Fig. 112. Presented values are averages over $3 \cdot 10^{4}$ (for $N=3 \cdot 10^{7}$ ) to $10^{5}$ (for $N=10^{6}$ ) samples. Although examined systems were rather large $\left(N_{\max }=3 \cdot 10^{7}\right)$, it is still difficult to make precise evaluation of exponents. Our results, $\xi=0.805(2)$ and $\gamma=0.195(2)$, satisfy Eq. (3), but we admit that the estimation of errors is based mainly on the visual inspection of data and the hope that further increase of the system size will not change much the final estimations. Although the difference is very small, such a value of $\xi$ seems to exclude the possibility $\xi=0.8$ obtained (exactly) for the $d=1$ annihilation process in the reaction-controlled limit. Our estimation of $\xi$ also slightly differs from previous molecular dynamics simulations result 0.785(5) made by Rey et al. [15] albeit with much smaller number of particles (they simulated systems with up to 262144 particles). Let us notice, that computing time in the search algorithm of Rey et al. increases as $O\left(N^{5 / 4} \ln (N)\right)$ while for the heap tree search (that we used) it increases only as $O(N \ln (N))$. Various computations based on the solution of the Boltzmann equation, and thus based on the molecular chaos hypothesis, predicts $\xi$ close to 0.77 [3, 7]. Disagreement between this result and our estimation shows that in $d=1$ Boltzmann kinetic theory has only limited validity.

To check for a possible multi-scaling we calculated the typical velocity $v^{\prime}(t)$ using the fourth-moment analogue of Eq. (1). Fig. [3]shows the exponent $\gamma^{\prime}$ that describes the decay of $v^{\prime}(t)$. Within our numerical precision $\gamma=\gamma^{\prime}$ and thus $v(t)$ as defined in Eq. (10) is the only characteristic velocity in this problem.

We also performed calculations for the singular initial velocity distribution with $\mu=1 / 2$. In this case we esti-

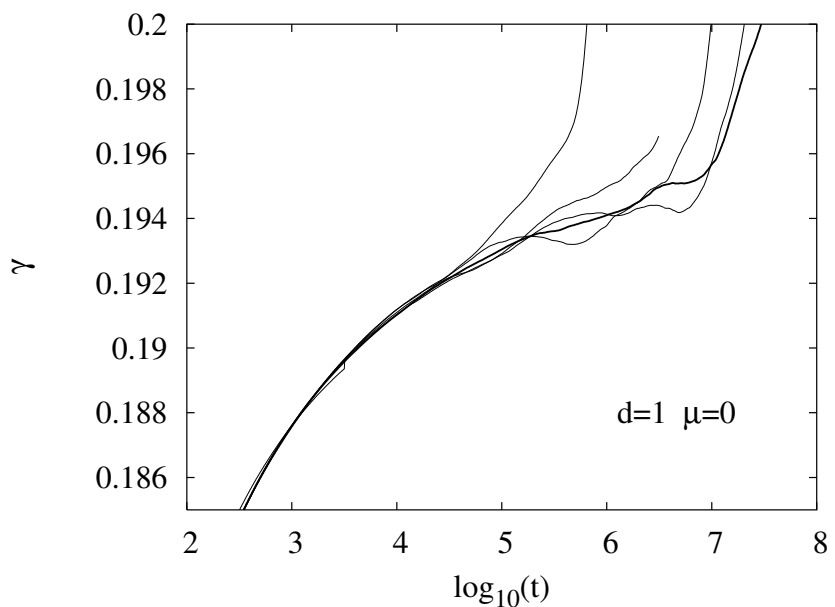

FIG. 2: The exponent $\gamma$ as a function of $t$ for $N=10^{6}, 5$. $10^{6}, 10^{7}, 2 \cdot 10^{7}$, and $3 \cdot 10^{7}$ (thick line). Initially, velocities have a gaussian distribution $(\mu=0)$.

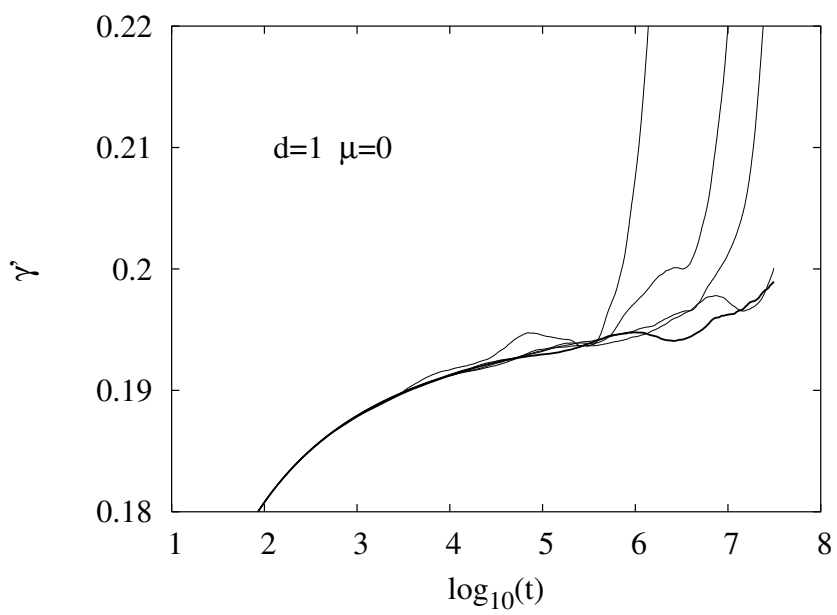

FIG. 3: The time dependence of the exponent $\gamma^{\prime}$ that describes the decay of the typical velocity defined via the fourth moment. Calculations were made for $N=10^{6}, 5 \cdot 10^{6}, 10^{7}, 2$. $10^{7}$, and $3 \cdot 10^{7}$ (thick line). Initially, velocities have a gaussian distribution $(\mu=0)$.

mate (see Figs. 45) $\xi=0.585(2)$ and $\gamma=0.415(2)$. This result satisfies Eq. (3) and improves over previous estimations of $\xi$ reported in the literature that range from 0.5 to $0.6[2,3,14]$.

In the one dimensional case topological constraints imply that for a given particle potential partners for a collision are only two of its nearest neighbours. In two dimensions this is no longer the case and in principle any pair of particles can collide. For large $N$ it leads to a prohibitively large number of potential collisions that should be examined but only very few of them will actually take place. To overcome this difficulty one can divide available space into sectors and look for potential collisions only within a sector or neighbouring sectors. More details on 


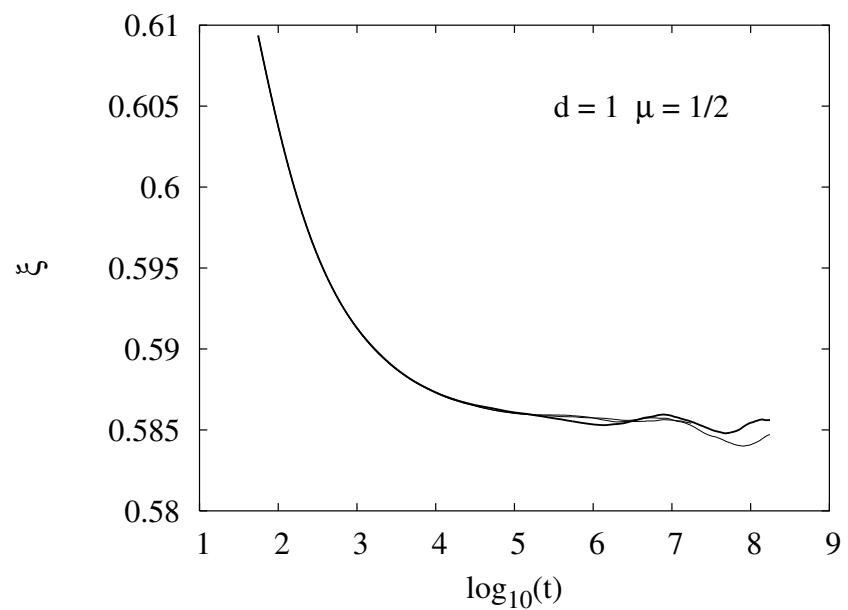

FIG. 4: The exponent $\xi$ as a function of $t$ for $N=10^{6}, 3 \cdot 10^{6}$, and $10^{7}$ (thick line). Initially, velocities have a distribution with $\mu=1 / 2$ singularity.

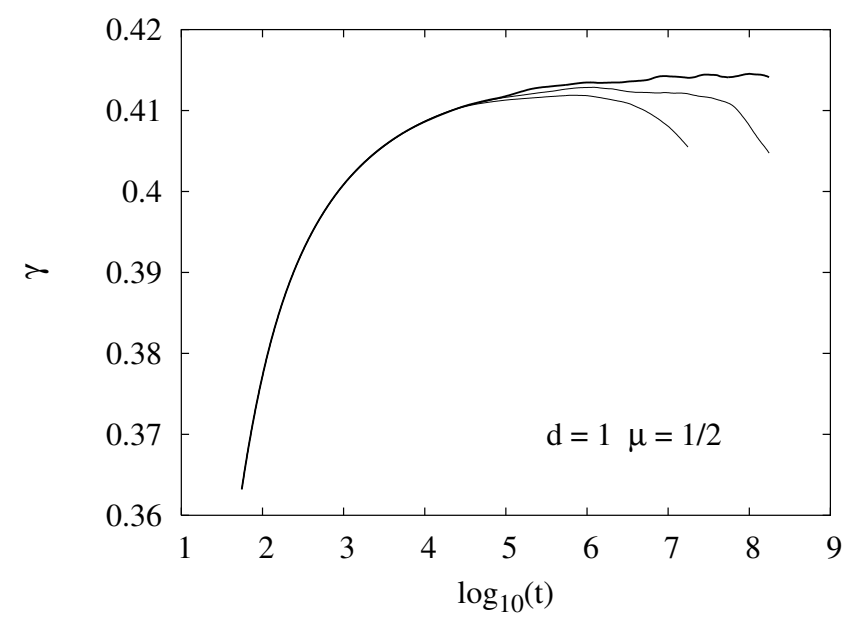

FIG. 5: The exponent $\gamma$ as a function of $t$ for $N=10^{6}, 3 \cdot 10^{6}$, and $10^{7}$ (thick line). Initially, velocities have a distribution with $\mu=1 / 2$ singularity.

this technique can be found elsewhere [11, 13].

For the gaussian initial velocity distribution results of our simulations are shown in Figs 6 7 In two dimensions size of particles $r$ relative to the linear system size $L$ becomes a relevant variable that might affect e.g., the time needed to reach asymptotic regime. Presented results are obtained for the packing fraction $f=\pi r^{2} N / L^{2}=0.0079$ but similar results were obtained for $f=0.031$. After averaging over $10^{3}-10^{4}$ samples, we estimate $\xi=0.872(2)$ and $\gamma=0.129(2)$. Such results satisfy Eq. (3) and are in a very good agreement with calculations based on the Boltzmann equation [3, 5], confirming thus validity of molecular chaos hypothesis in this case. Let us notice that our estimation of $\xi$ definitely excludes the reactioncontrolled value $\xi=8 / 9$. Moreover, the maximal number of particles examined in our approach $\left(9 \cdot 10^{6}\right)$ is almost

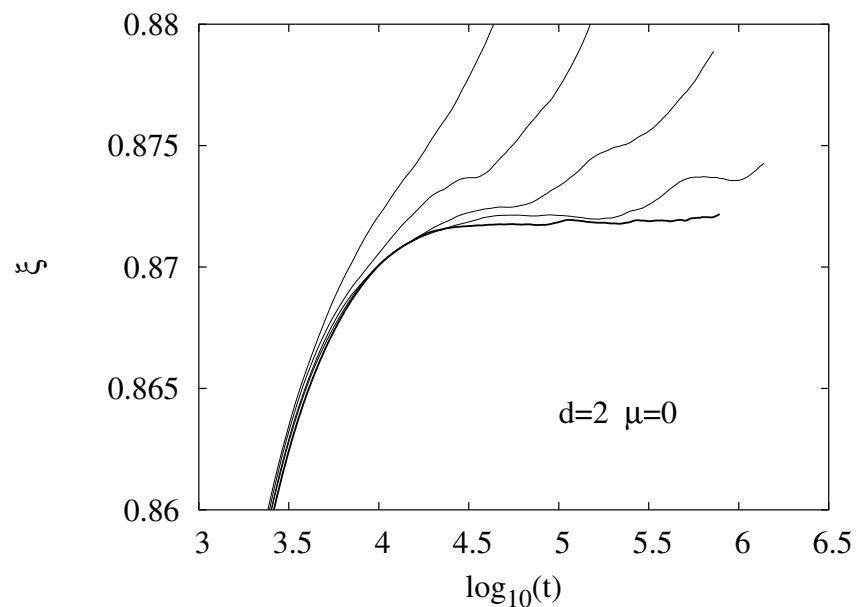

FIG. 6: The exponent $\xi$ as a function of $t$ for $N=$ $300^{2}, 500^{2}, 1000^{2}, 2000^{2}$, and $3000^{2}$ (thick line). Initially, velocities have a gaussian distribution $(\mu=0)$.

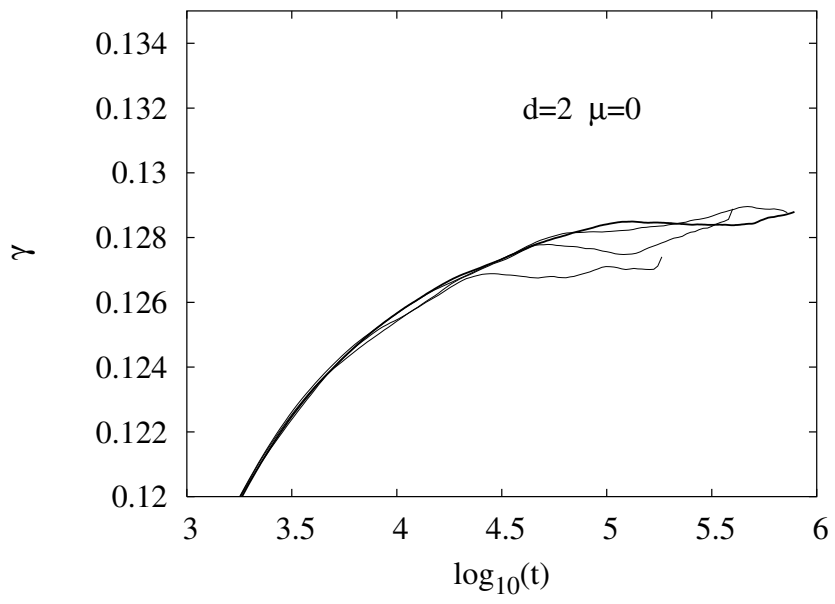

FIG. 7: The exponent $\gamma$ as a function of $t$ for $N=$ $300^{2}, 500^{2}, 1000^{2}$, and $3000^{2}$ (thick line). Initially, velocities have a gaussian distribution $(\mu=0)$.

20 times larger that for previously reported $d=2$ eventdriven simulations [5].

We also made calculations for the $\mu=1 / 2$ case and the results are presented in Fig. 89 We estimate $\xi=$ $0.83(1)$ and $\gamma=0.17(1)$. Similar value of $\xi$ was recently obtained using numerical integration of the Boltzmann equation [3]. In Table \we collect all our final results.

In conclusion, implementing a heap-tree search algorithm and sectorization we made extensive event-driven molecular dynamics simulations of the ballistic annihilation. Obtained estimations of decay exponents $\xi$ and $\gamma$ obey the scaling relation $\xi+\gamma=1$ and improve over previously reported results. Our calculations suggest that the typical velocity as defined via the second moment sets the only characteristic scale in the problem. Very good agreement of our simulations with predictions of 


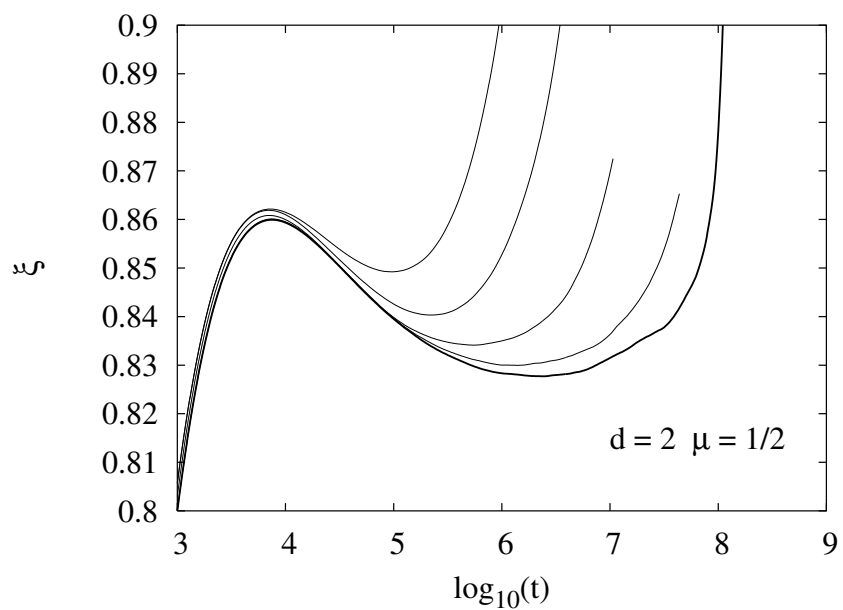

FIG. 8: The exponent $\xi$ as a function of $t$ for $N=$ $300^{2}, 500^{2}, 1000^{2}, 2000^{2}$, and $3000^{2}$ (thick line). Initially, velocities have a distribution with $\mu=1 / 2$ singularity.

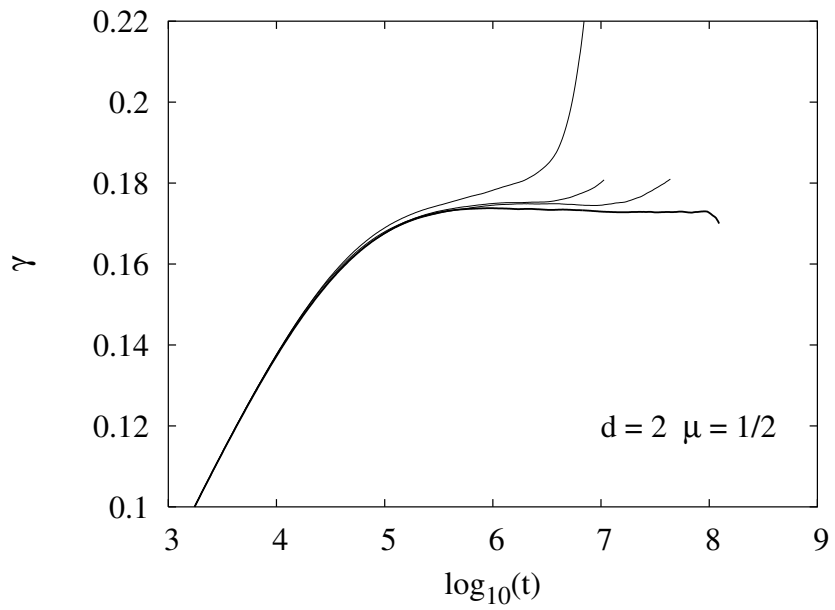

FIG. 9: The exponent $\gamma$ as a function of $t$ for $N=$ $500^{2}, 1000^{2}, 2000^{2}$, and $3000^{2}$ (thick line). Initially, velocities have a distribution with $\mu=1 / 2$ singularity.

\begin{tabular}{|c|c|c|c|c|c|c|}
\hline $\mathrm{d}$ & $\mu$ & $\xi$ & $\gamma$ & $\xi[14]$ & $\xi[4]$ & $\xi[3]$ \\
\hline \multirow{2}{*}{1} & 0 & $0.805(2)$ & $0.195(2)$ & 0.666 & 0.8 & 0.769 \\
\cline { 2 - 7 } & $1 / 2$ & $0.585(2)$ & $0.415(2)$ & 0.5 & - & 0.6 \\
\hline 2 & 0 & $0.872(2)$ & $0.129(2)$ & 0.8 & 0.75 & 0.87 \\
\cline { 2 - 7 } & $1 / 2$ & $0.83(1)$ & $0.17(1)$ & 0.75 & - & 0.84 \\
\hline
\end{tabular}

TABLE I: Exponents $\xi$ and $\gamma$ as calculated in the present paper. Estimations of $\xi$, based on the analysis of the Boltzmann equation [3, 14] and in the reaction-controlled limit [4] are presented in the last three columns.
Boltzmann kinetic theories in the $d=2$ case confirms validity of the latter approach. In the $d=1$ case small differences between results of these two approaches exist. It would be interesting to extend our approach to some other model phenomena whose kinetics is still not fully understood as e.g., ballistic aggregation [4, 16] or probabilistic ballistic annihilation [17].

Acknowledgments: A. Lipowski acknowledges the research grant 1 P03B 01427 from KBN. A. L. Ferreira acknowledges financial support from Fundação Ciência e Tecnologia (FCT) of Portugal.
[1] F. Leyvraz, Phys. Rep. 383, 95 (2003).

[2] E. Ben-Naim, S. Redner, and F. Leyvraz, Phys. Rev. Lett. 70, 1890 (1993).

[3] E. Trizac, Phys. Rev. Lett. 88, 160601 (2002).

[4] E. Trizac and P. L. Krapivsky, Phys. Rev. Lett. 91, 218302 (2003).

[5] J. Piasecki, E. Trizac, and M. Droz, Phys. Rev. E 66, 066111 (2002).

[6] B. Chopard, A. Masselot, and M. Droz, Phys. Rev. Lett. 81, 1845 (1998).

[7] P. L. Krapivsky and C. Sire, Phys. Rev. Lett. 86, 2494 (2001).

[8] G. F. Carnevale, Y. Pomeau, and W. R. Young, Phys. Rev. Lett. 64, 2913 (1990). L. Frachebourg, Phys. Rev. Lett. 82, 1502 (1999).
[9] D. C. Rapaport, The Art of Molecular Dynamics Simulations, (Cambridge University Press, Cambridge, 1995).

[10] D. E. Knuth, The Art of Computer Programming, (Addison-Wesley, Reading, MA, 1968).

[11] B. D. Lubachevsky, J. Comput. Phys. 94, 255 (1991).

[12] M. Marín and P. Cordero, Comput. Phys. Commun. 92, 214 (1995).

[13] S. Miller and S. Luding, J. Comput. Phys. 193, 306 (2004).

[14] E. Ben-Naim, P. L. Krapivsky, F. Leyvraz, and S. Redner, J. Phys. Chem. 98, 7284 (1994).

[15] P.-A. Rey, M. Droz, and J. Piasecki, Phys. Rev. E 57, 138 (1998).

[16] E. Trizac and J.-P. Hansen, Phys. Rev. Lett. 74, 4114 (1995). 
[17] F. Coppex, M. Droz, and E. Trizac, Phys. Rev. E 72 021105 (2005). 